\title{
Atuação da fisioterapia aquática funcional no deslocamento na postura sentada na amioplasia congênita: estudo de caso
}

\author{
Performance of functional aquatic physical therapy on shift in sitting posture \\ in congenital amyoplasia: case study
}

Renata D'Agostini Nicolini-Panisson', Ricardo Cristian Hengles², Deborah Cristina Garcia de Mattos ${ }^{2}$

${ }^{1}$ Fisioterapeuta do Instituto de Neuro-Ortopedia e professora da Faculdade da Serra Gaúcha, Caxias do Sul, RS.

${ }^{2}$ Fisioterapeutas do setor de fisioterapia aquática da Unidade Central Ibirapuera/Associação de Assistência à Criança Deficiente (AACD), São Paulo, SP.

\section{RESUMO}

Objetivos: Descrever a atuação da fisioterapia aquática funcional no arrastar na postura sentada em uma criança com amioplasia congênita.

Descrição do Caso: Uma menina de três anos de idade, portadora de amioplasia congênita, participou de um programa de reabilitação composto por 24 sessões de fisioterapia aquática. As avaliações foram realizadas no primeiro dia, após 12 sessões e ao final do programa. Foram mensurados o tempo de arrastar na postura sentada e os itens 24 (sentada no tapete mantém braços livres por três segundos) e 25 (sentada no tapete toca brinquedo pequeno na frente), da dimensão sentar do instrumento Gross Motor Function Measure 88 (GMFM-88). O tratamento foi um programa de exercícios com objetivo de ativação muscular, flexibilidade, transferência de peso e estabilização de tronco na postura sentada. Após a intervenção de fisioterapia aquática, verificou-se melhora da função motora, com maior velocidade de deslocamento na posição sentada.

Conclusões: Verificaram-se resultados positivos após o programa de fisioterapia aquática aplicada a essa paciente com amioplasia congênita. Observou-se evolução no controle de tronco, melhorando sua estabilidade e repercutindo em sua funcionalidade, com aumento da velocidade no arrastar na postura sentada.

DESCRITORES: AMIOPLASIA CONGÊNITA; ARTROGRIPOSE; HIDROTERAPIA; REABILITAÇ̃̃O.

\section{ABSTRACT}

Aims: To describe the role of aquatic therapy in drag on sitting posture in a child with congenital amyoplasia.

Case Report: A three-year-old girl with congenital amyoplasia participated in a rehabilitation program consisting of 24 sessions of aquatic therapy. The evaluations were performed on the first day, after 12 sessions and at the end of the program. The time of drag in the seated position and the items 24 (sitting on the mat keeps arms free for three seconds) and 25 (sitting on the carpet plays small toy in front) of the sitting dimension of the instrument Gross Motor Function Measure 88 (GMFM-88) were measured. The therapy consisted of an exercise program with the goals of muscle activation, flexibility, weight transfer and trunk stabilization in the seated position. After the aquatic therapy intervention there was improvement in motor function, with greater displacement speed in the sitting position.

Conclusions: Following the aquatic therapy program applied to this patient with congenital amyoplasia, positive results were verified. There was improvement in trunk control, increasing its stability and reflecting on its functionality, with greater speed in drag in the sitting posture.

KEY WORDS: AMYOPLASIA CONGENITA; ARTHROGRYPOSIS; HYDROTHERAPY; REHABILITATION. 


\section{INTRODUÇÃO}

A artrogripose múltipla congênita é uma síndrome clínica presente ao nascimento, caracterizada por diversas contraturas articulares não progressivas. É uma patologia relativamente frequente, estimando-se que ocorra em um para cada 3.000 nascidos vivos. Pode assumir diversas formas, dependendo dos membros acometidos e da gravidade do comprometimento. A forma mais comum de artrogripose é a amioplasia congênita, que corresponde a cerca de um terço de todos os casos. A amioplasia caracteriza-se pelo acometimento predominante dos membros, com falta de movimento nas articulações, podendo levar ao subdesenvolvimento ou ausência do tecido muscular. ${ }^{1-3}$

A força muscular precária e as contraturas têm um impacto importante no desempenho da função motora de crianças com amioplasia. ${ }^{4}$ Dependendo do grau de acometimento dos membros, essas crianças apresentarão níveis funcionais diferentes, variando de crianças deambuladoras a não deambuladoras. ${ }^{4} \mathrm{O}$ arrastar na postura sentada é uma forma encontrada por crianças não deambuladoras para realizarem um deslocamento e serem funcionais. ${ }^{5}$ Cada criança realizará essa tarefa de maneira diferente, dependendo de suas deformidades e musculatura ativa. É importante estimular as várias formas de deslocamento, visando o movimento independente com maior exploração e alcance de brinquedos, além de facilitar a integração com a família e com outras crianças. ${ }^{6}$

A reabilitação deve ter inicio precoce e o paciente precisa receber acompanhamento com uma equipe interdisciplinar. $\mathrm{O}$ ambiente aquático proporciona facilitações devido às propriedades físicas da água, auxiliando o treino da função do arrastar na postura sentada. ${ }^{7}$ A associação de conceitos atuais da Classificação Internacional de Funcionalidade (CIF), do controle motor, da tarefa voltada à função e, principalmente, do uso de indicadores de evolução, favorecem bons resultados com a fisioterapia aquática. ${ }^{7}$ A correta determinação de objetivos funcionais mensuráveis é imprescindível para que os ganhos obtidos em meio líquido sejam transferidos para o solo, e portanto traduzidos em ganhos funcionais. ${ }^{7}$

Os exercicíos realizados na água auxiliam no desenvolvimento do equilíbrio, coordenação e postura. As atividades na água proporcionam melhora na circulação sanguínea, garantindo adequada nutrição dos tecidos. ${ }^{8}$

Até o presente momento, são escassos os estudos encontrados a respeito da reabilitação funcional na amioplasia congênita e não existem estudos com o uso de fisioterapia aquática nessa condição. Sendo assim, o objetivo deste estudo foi descrever a atuação da fisioterapia aquática funcional na função de arrastar na postura sentada em uma criança com amioplasia congênita.

\section{RELATO DO CASO}

Este estudo foi realizado no setor de fisioterapia aquática da Associação de Assistência à Criança Deficiente (AACD) em São Paulo, SP. O estudo foi aprovado pelo Comitê de Ética em Pesquisa da AACD sob o parecer consubstanciado de número 506.220 e os responsáveis assinaram um termo de consentimento livre e esclarecido.

O sujeito da pesquisa foi uma criança do sexo feminino, com três anos de idade, com contraturas articulares simétricas envolvendo os membros inferiores e superiores. Apresentava deformidade em valgo de joelhos, pés equino cavo varo, ombros em rotação interna e adução, cotovelos em extensão, punhos e polegares levemente fletidos e polegar esquerdo aduzido.

A fisioterapia aquática funcional foi realizada em uma piscina coberta e aquecida com temperatura média da água de $32^{\circ} \mathrm{C}$. As sessões foram realizadas uma vez por semana, com duração de 35 minutos, totalizando 24 sessões. O programa de exercícios incluiu alongamentos musculares de flexores de quadril, extensores de joelho e plantiflexores; ativação muscular de abdominais profundos e superficiais, glúteo máximo e médio; treino dos componentes da função do deslocamento na postura sentada, como estabilização de tronco, dissociação de cinturas e transferência de peso na postura sentada; além do treino da função em diferentes profundidades de água e utilizando a inclinação da própria piscina, facilitando e posteriormente dificultando a atividade. Foram utilizados brinquedos para tornar mais lúdicos os atendimentos, como bolinhas de plástico, pranchas e brinquedos de borracha.

A paciente foi submetida às avaliações no pré e no pós-protocolo. O arrastar na postura sentada foi avaliado antes da intervenção, após 12 sessões e ao final de 24 sessões. Utilizou-se a mensuração do tempo de deslocamento na postura sentada em um tablado de 2 metros com um cronômetro digital. $\mathrm{O}$ resultado considerado foi o melhor desempenho após três tentativas.

Como instrumentos de avaliação foram utilizados dois itens do Gross Motor Function Measure 88 (GMFM-88), que tem o objetivo de avaliar mudanças na função motora grosseira de crianças. ${ }^{9}$ 
O GMFM-88 consiste em 88 itens agrupados em cinco dimensões: (A) deitar e rolar, (B) sentar, (C) engatinhar e ajoelhar, (D) ficar em pé e (E) andar, correr e pular. Cada item é avaliado em uma escala de quatro pontos ( 0 - não inicia; 1 - inicia; 2 - parcialmente completa; e 3 - completa), sendo que altos escores indicam melhor função motora grosseira. Cada tarefa foi pontuada de acordo com as instruções contidas no Manual do GMFM-88. ${ }^{6}$ Nesta pesquisa utilizaram-se os itens 24 (sentada no tapete mantém braços livres por 3 segundos) e 25 (sentada no tapete com um brinquedo pequeno na frente inclinase para a frente, toca o brinquedo, endireita-se sem apoio do braço) da dimensão sentar, visando avaliar o controle de tronco. A avaliação foi realizada por uma fisioterapeuta com treinamento para a aplicação do GMFM-88 e com experiência no uso da medida. Para a realização dos testes, a criança estava vestida com o mínimo de roupa possível e sem calçados.

Para análise dos dados foi utilizada estatística descritiva, com demonstração do melhor valor obtido durante as avaliações e porcentagem para verificar os dados pré e pós-atendimento.

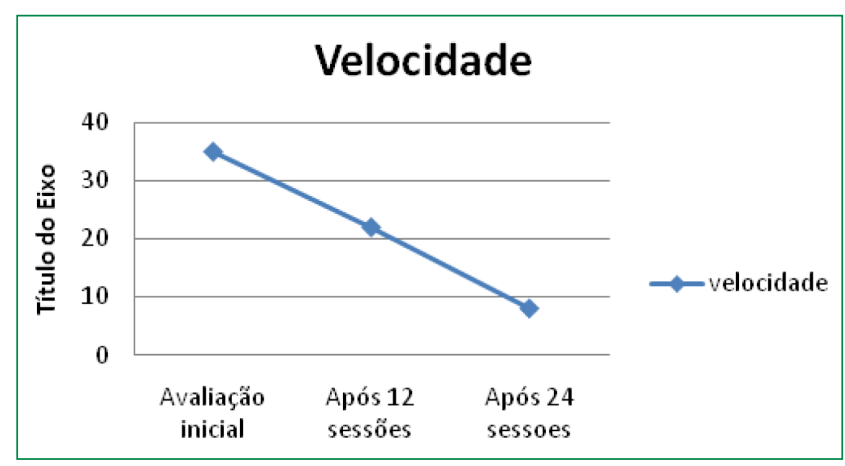

Figura 1. Valores de tempo, em segundos, do deslocamento na postura sentada em um percurso de 2 metros. Avaliação inicial, após 12 sessões de fisioterapia aquática e após 24 sessões.

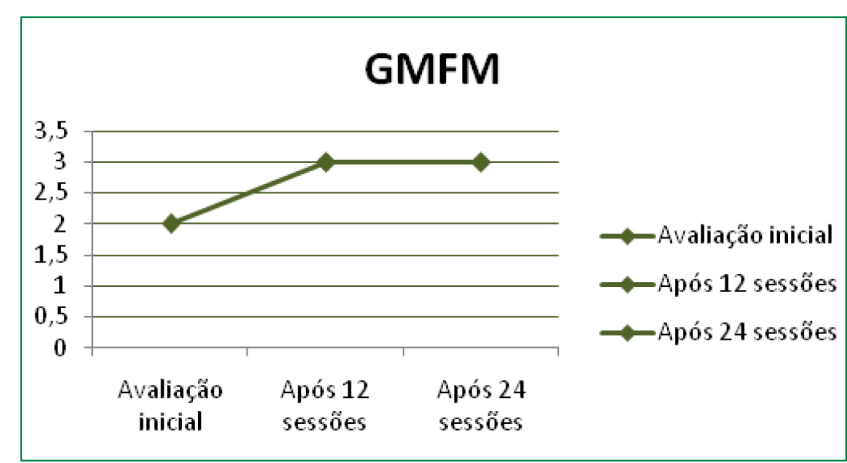

Figura 2. Valores do escore do GMFM (item 24 e 25). Préintervenção, após 12 sessões e após 24 sessões de fisioterapia aquática.
Após a intervenção específica da fisioterapia aquática funcional, observou-se uma diminuição no tempo de deslocamento na postura sentada. Inicialmente o tempo foi de 35 segundos para percorrer os 2 metros do tablado; com 12 sessões observou-se a diminuição do tempo para 22 segundos e, ao final das 24 sessões, o tempo foi de 8 segundos (Figura 1).

Nos itens 24 e 25 do GMFM- 88 houve um aumento do escore inicial que era 2 (parcialmente completa) para 3 (completa) no meio da intervenção com 12 sessões, o qual se manteve até o final das 24 sessões (Figura 2).

\section{DISCUSSÃO}

O programa de exercícios relatado seguiu a metodologia preconizada pelo novo conceito de trabalho da funcionalidade no meio aquático. ${ }^{7}$ O primeiro passo após a avaliação específica do indivíduo é a determinação do objetivo funcional, que é a função em si, e dos objetivos específicos, que são os componentes da função. No momento em que determinamos os objetivos, levamos em conta todos os aspectos do diagrama da Classificação Internacional de Funcionalidade, Incapacidade e Saúde (CIF): funções e estruturas do corpo, atividade, participação, fatores ambientais e fatores pessoais, e não somente a condição de saúde. Os potenciais de cada indivíduo devem ser levados em conta para avaliar a sua participação. ${ }^{10}$

Segundo Silva e Branco 7 , a correta escolha destes objetivos consegue direcionar o tratamento e atingir a função com a máxima qualidade, considerando os limites do indivíduo e o seu ambiente. O uso de indicadores de evolução quantitativos, com testes mensuráveis e qualitativos, como a filmagem, facilita a avaliação periódica dos indivíduos. ${ }^{7}$

$\mathrm{O}$ objetivo funcional determinado para esta paciente foi aprimorar o arrastar na postura sentada como forma de deslocamento domiciliar. Os objetivos específicos foram o estímulo dos componentes dessa função, como descritos por Relvas et al.: ${ }^{5}$ estabilidade de tronco e transferências de peso na postura sentada. Sendo que a estabilidade de tronco busca uma estabilização dinâmica: estimula o aumento dos limites de estabilidade laterais, com deslocamentos da linha de gravidade no limite da base de suporte na postura sentada; treina as reações de equilibrio, endireitamento e proteção; e otimiza as forças internas que atuam nesta estabilidade. ${ }^{5}$

Segundo a CIF, nesse processo dinâmico a funcionalidade da criança depende de contínuas interações com a família ou com outros cuidadores, 
não podendo ser vista isoladamente, mas sim em um contexto familiar. A influência familiar sobre a funcionalidade da criança é maior nesta fase de desenvolvimento. A família, considerada como um fator ambiental pode dificultar o processo, caso seja superproterora ou não siga as orientações, ou pode facilitar, quando segue as recomendações e auxilia a equipe. ${ }^{7,10}$ Corroborando com a CIF, a criança e a família neste caso estiveram participativas no processo de reabilitação, seguindo todas as orientações. No ambiente domiciliar não havia nenhuma barreira arquitetônica que impedisse o treino da função.

A artrogripose caracteriza-se por contraturas musculares e limitações em diversas articulações, por graus variados de fibrose muscular e pelo espessamento e encurtamento da cápsula periarticular e dos tecidos ligamentares das articulações afetadas. A fraqueza muscular e a rigidez articular dificultam a correção das deformidades congênitas, porém o prognóstico é melhor quando o tratamento é instituído precocemente. ${ }^{11}$

Embora não existam estudos que utilizam o deslocamento sentado como forma de deslocamento nesses pacientes, diversos autores citam que a reabilitação na amioplasia congênita atua com o principal objetivo de estimular o desenvolvimento motor global da criança, visando ao máximo sua dinâmica funcional. ${ }^{12-13}$ No caso relatado, a partir do quadro motor apresentado pela paciente considerando a sua principal forma de deslocamento, conseguiu-se maximizar a sua função.

As variações no ambiente aquático, como a turbulência e o empuxo, favorecem um trabalho de equilíbrio estático e dinâmico. $\mathrm{O}$ empuxo diminui a ação da musculatura antigravitacional para a manutenção da postura, trabalhando a musculatura com maior facilidade que em solo, respeitando o comprometimento motor. $\mathrm{O}$ mesmo associado a manuseios: a pressão hidrostática e a turbulência facilitam as respostas automáticas de equilíbrio, estáticas ou dinâmicas, e auxiliam na melhora da qualidade funcional da postura e no movimento, por meio da ativação de um mecanismo postural mais próximo do normal. ${ }^{14-16}$

Após a intervenção, com a diminuição do tempo no deslocamento sentado de 35 para 8 segundos, a criança ficou mais participativa em suas atividades, deslocando-se sentada com mais eficiência e rapidez, conseguindo passar de um cômodo para outro, em sua residência, de forma independente. $\mathrm{O}$ recurso terapêutico da água favoreceu um meio lúdico, que facilitou a aprendizagem do arrastar sentado, fazendo a criança ir atrás de brinquedos ou até mesmo realizar brincadeiras de competição. ${ }^{17} \mathrm{O}$ tratamento em meio aquático torna o ambiente agradável e divertido, sendo um fator importante em doenças que necessitam de um longo período de reabilitação, como é o caso da amioplasia congênita. $\mathrm{O}$ caráter lúdico estimula o maior envolvimento da criança com a terapia, otimizando os resultados. ${ }^{18}$

Este relato sugere que a fisioterapia aquática funcional seja eficaz no aprimoramento da função de arrastar na postura sentada em uma criança com amioplasia congênita. Verificaram-se resultados positivos após o programa de fisioterapia aquática. Observou-se melhora no controle de tronco aumentando assim a estabilidade nesta postura, repercutindo em sua funcionalidade, com melhora da velocidade no arrastar na postura sentada.

Não se tem conhecimento de pesquisas com o uso de fisioterapia aquática na reabilitação funcional de crianças com amioplasia congênita. Com a evolução científica atual e as mudanças nos conceitos e atuações fisioterapêuticas, é imprescindível que os fisioterapeutas realizem seus tratamentos cada vez mais embasados em evidências, tendo bons indicativos de evolução tanto qualitativos como quantitativos, pelo que se recomenda que sejam feitos estudos que possam confirmar estes resultados.

\section{REFERÊNCIAS}

1. Hall JG, Reed SD, Driscoll EP. Part I. Amyoplasia: a common, sporadic condition with congenital contractures. Am J Med Genet. 1983;15(4):571-90.

2. Hall JG, Reed SD, McGillivray BC, et al. Part II. Amyoplasia: twinning in amyoplasia specific type of arthrogryposis with an apparent excess of discordantly affected identical twins. Am J Med Genet. 1983;15(4):591-9.

3. Bernstein RM. Arthrogryposis and amyoplasia. J Am Acad Orthop Surg. 2002;10(6):417-24.

4. Kroksmark AK, Kimber E, Jerre R, Beckung E, Tulinius M. Muscle involvement and motor function in amyoplasia. Am J Med Genet. Part A. 2006;140(16):1757-67.

5. Relvas PCA, Nicolini RD, Sousa PPC. Fisioterapia aquática em pacientes com malformações congênitas. In: Silva JB, Branco F. Fisioterapia Aquática Funcional. São Paulo: Artes Médicas; 2011. p. 275-304. 
6. Russell D, Rosenbaum P, Cadman D, Gowland C, Hardy S, Jarvis S. The Gross Motor Function Measure: a means to evaluate the effects of physical therapy. Dev Med Child Neurol. 1989;31(3):341-52.

7. Silva JB, Branco F. Fisioterapia aquática funcional e seus indicadores de qualidade. In: Silva JB, Branco F. Fisioterapia aquática funcional. São Paulo: Artes Médicas; 2011. p. 19-21.

8. Carvalho RL, Santos CE. Efeito da imersão associada à cinesioterapia na artrogripose: Rev Cient UNIFAE. 2008:2(1):10-4.

9. Russell D, Rosenbaum P, Growland C, Hardy S, Plews N, et al. Administration and scoring. In: University M, editor. Gross Motor Function Mesuare Manual. 2nd ed. Toronto; 1993. p. 1-125.

10. CIF-CJ: Classificação Internacional de funcionalidade, incapacidade e saúde: versão crianças e jovens. São Paulo: Edusp; 2011.

11. Shepherd RB. Fisioterapia em Pediatria. 3ํㅡㄹ. São Paulo: Santos, 1996.

12. Richards S. Atualização em Conhecimento Ortopédico. Pediatria. São Paulo. Atheneu, 2002.

13. Risarde SMA. Artrogripose múltipla congênita. Rio de Janeiro. 2007.

14. Caromano FA, Themudo MRFF, Candeloro JM. Efeito Fisiológico da Imersão e do Exercício na Água. Fisiot Brasil. 2003;4(1):60-5.

15. Marins RS. Síndrome de Down e terapia aquática: possibilidades da infância dos efeitos físicos da água na musculatura estriada esquelética e na postura. Reabilitar. 2001;10(1):12-20.

16. Carregaro RL, Toledo AM. Efeitos fisiológicos e evidências científicas da eficácia da fisioterapia aquática. Rev Movimenta. $2008 ; 1(1): 23-7$.

17. Costa FAS. Recreação aquática: o lúdico do brincar e psicomotricidade na água. EFDeportes Rev Digital. 2010;15(146):1.

18. Ruoti RG, Morris DM, Cole AJ. Reabilitação Aquática. São Paulo: Manole; 2000. 